This is a postprint version of the following published document:

P.J. Pinzón, I. Pérez, J. M. S. Pena, C. Vázquez (2014). "Spectral method for fast measurement of twisted nematic liquid crystal cell parameters". In Applied Optics, 53 (2014) 23, pp. 5230-5237. Available in http://dx.doi.org/10.1364/AO.53.005230

(c) 2014 Optical Society of America 


\title{
Spectral method for fast measurement of twisted nematic liquid crystal cell parameters
}

\author{
Plinio Jesús Pinzón, ${ }^{\star}$ Isabel Pérez, José Manuel Sánchez-Pena, and Carmen Vázquez \\ Group of Displays and Photonics Applications, Electronic Technology Department, \\ Universidad Carlos III de Madrid, C/Butarque 15, 28911 Leganes, Spain \\ ${ }^{\star}$ Corresponding author: ppinzon@ing.uc3m.es
}

\begin{abstract}
We present an experimental approach for the fast measurement of twisted nematic (TN) liquid crystal (LC) cells' parameters. It is based on the spectral measurements of the light transmitted by the system polarizer-reference wave plate-LC cell-analyzer. The cell parameters are obtained by fitting the theoretical model to the experimental data. This method allows determining the rubbing angle, the twist angle and its sense, and the spectral dispersion of the LC cell retardation, simultaneously, with few measurements and without the need of applying voltage or any specific analytical conditions. The method is validated by characterizing two different TN cells with retardations of about 0.91 and $1.85 \mu \mathrm{m}$. The birefringence relative error is less than $1.3 \%$.
\end{abstract}

OCIS codes: (230.3720) Liquid-crystal devices; (230.6120) Spatial light modulators; (260.1440) Birefringence; (120.4630) Optical inspection.

\section{Introduction}

Twisted nematic (TN) liquid crystal (LC) devices are used extensively nowadays in multiple applications like information displays; spatial light modulators for optical correlators, projection systems, and laser beam steering; or optical communication devices such as optical switches and wavelength tunable filters [1,2].

The optical characteristics and performance of a TN device depend significantly on four cell parameters [ $\underline{3}-\underline{5}$ ]: the molecular twist angle $\left(\phi_{T}\right)$, the rubbing direction angle at the input surface $\left(\alpha_{T}\right)$, the cell 31 thickness $\left(d_{T}\right)$, and the LC material birefringence $(\Delta n)$, which depends on the wavelength $(\lambda)$. There- fore, there is a constant need for accurate characterization of the LC cell parameters during themanufacturing processes. Several characterization methods have been proposed in the past. They are typically classified as single wavelength methods or spectral methods.
Single wavelength methods, as a rule, are more reliable in an experiment. However, they are able to determine the cell retardation $\left(d_{T} \times \Delta n\right)$ for a fixed wavelength only. Therefore, they are inherently unable to determine the wavelength dispersion of $\Delta n$, $\Delta n(\lambda)$. The most common single wavelength method for characterizing TN cells was proposed by Soutar and $\mathrm{Lu}$ [6]. It consists of the nonlinear fitting of a theoretical model, based on the Jones matrix calculus. The transmittances of the TN cell between parallel and crossed polarizers are measured at a fixed $\lambda$, and at different output polarizer (analyzer) angles $\alpha_{A}$, between $0^{\circ}$ and $360^{\circ}$. This is an effective method, but it is not able to determine unequivocally the twist sense and the rubbing direction angle, due to the mathematical ambiguities of its theoretical model. Many single wavelength methods have been proposed to find a unique answer for $\phi_{T}$ and $\alpha_{T}$ [7-13]. However, they require multiple rotation angles for the polarizers or the LC cell, additional measurements with different voltage levels, with 
polarimeters, or the application of specific analytical conditions.

On the other hand, spectral methods allow us to determine $\Delta n(\lambda)$, even considering the transmission ripple of the LC devices caused by the multiple reflections in the indium tin oxide layers deposited on the inner cell surfaces [14], overcoming this important limitation of single wavelength methods. However, the spectral methods reported to date are not able to determine $\phi_{T}$, the $\phi_{T}$ sense, $\alpha_{T}$, and $d_{T} \times \Delta n(\lambda)$ simultaneously [14-17]. This is because, in general, most of the characterization methods proposed to date are oriented to display applications, where the determination of $\left|\phi_{T}\right|, \alpha_{T}$, and $\Delta n(\lambda)$ is enough. However, the performance of the optical communication devices based on stacked structures of LC cells [18-21] is very sensitive to the $\phi_{T}$ sense as well as the other parameters.

In this work, a new spectral method for the precise determination of TN cell parameters is presented. The method consists of measuring the transmittance, between parallel and crossed polarizers, of a reference wave plate, $\mathrm{H}$, with well-known characteristics, in series with the TN sample. Optical transmittances are measured rotating $\mathrm{H}$ for different azimuth angles, $\alpha_{H}$ (only three are needed), in the optical spectrum of interest. Then, the TN cell parameters are determined by fitting the theoretical model to the experimental curves. The model is obtained with the Jones matrix calculus and the extended Cauchy model (for the LC birefringence dispersion calculations). The curve fitting is done by implementing a genetic algorithm (GA) with wide searching limits and without specific initial conditions. This procedure allows us to determine the rubbing angle $\alpha_{T}$, the twist angle $\phi_{T}$, and its sense, as well as the dispersion of the LC retardation, $d_{T} \times \Delta n(\lambda)$, simultaneously, with few measurements, and using a simple procedure.

\section{Modeling of Nematic LC Cells}

LC devices are usually modeled with Mueller [13] or Jones matrices [3]. Unlike a Mueller matrix, which is suitable for all kinds of optical systems, a Jones matrix can only be applied to a nondepolarizing optical system. Then, the Mueller-matrix-based models of LC devices provide a more accurate representation of the polarization state of the transmitted light than the Jones-matrix-based models. However, Jonesmatrix-based models generally provide a good compromise between physical adequacy and accuracy with computational efficiency and optimization facilities in the theoretical model [22]. Moreover, the characterization methods that use Jones matrix models are less complex and require fewer measurements (less input polarization states), so they are widely used $[\underline{6}-\underline{10}]$.

\section{A. Jones Matrix Representation}

Figure 1 shows the scheme of a TN cell. It can be modele $\bar{d}$ as a stack of $N$ nematic homogeneous

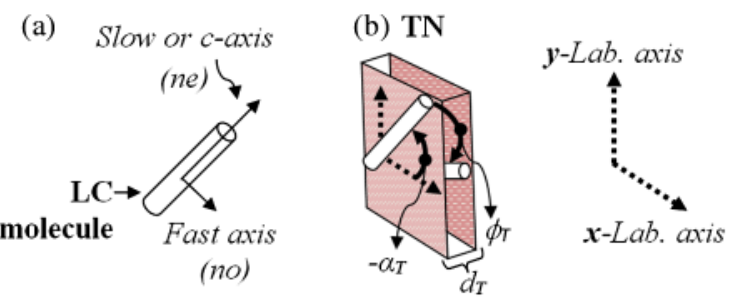

Fig. 1. (a) Nematic LC molecule and (b) twisted nematic LC cell (TN) representation with respect to the $x-y$ laboratory axes.

layers, each one with a constant orientation angle of the molecular axis ( $c$ axis) with respect to the $x$ axis, varying in a total twist angle $\phi_{T}$, in increments of $\Delta \phi_{T}=\phi_{T} / N$. Therefore, the Jones matrix of a TN cell [므, 23] with its front $c$ axis at $\alpha_{T}$ and total twist $\phi_{T}$, with respect to the $x-y$ laboratory axes, is given by

$$
\begin{aligned}
W_{T}\left(\alpha_{T}\right)= & R^{-1}\left(\alpha_{T}\right) \times\left[\begin{array}{cc}
\cos \left(\phi_{T}\right) & -\sin \left(\phi_{T}\right) \\
\sin \left(\phi_{T}\right) & \cos \left(\phi_{T}\right)
\end{array}\right] \\
& \times\left[\begin{array}{ll}
\cos (X)-i \frac{\Gamma_{T}}{2} \frac{\sin (X)}{X} & \phi_{T} \frac{\sin (X)}{X} \\
-\phi_{T} \frac{\sin (X)}{X} & \cos (X)+i \frac{\Gamma_{T}}{2} \frac{\sin (X)}{X}
\end{array}\right] \\
& \times R\left(\alpha_{T}\right),
\end{aligned}
$$

where $X=\left(\phi_{T}^{2}+\Gamma_{T}^{2} / 4\right)^{1 / 2}$ and $\Gamma_{T}$ is the phase delay, produced by the birefringence $\left[\Delta n=n_{e}-n_{o}\right.$; see Fig. 1(a)] of the LC molecules, between the polarization components of a light beam, at a specific wavelength $\lambda$. This phase delay is given by

$$
\Gamma_{T}=2 \pi \frac{\Delta n d_{T}}{\lambda} .
$$

$R\left(\alpha_{T}\right)$ is the rotation matrix of the $c$ axis orientation angle with respect to the $x$ axis:

$$
R\left(\alpha_{T}\right)=\left[\begin{array}{cc}
\cos \left(\alpha_{T}\right) & \sin \left(\alpha_{T}\right) \\
-\sin \left(\alpha_{T}\right) & \cos \left(\alpha_{T}\right)
\end{array}\right]
$$

Hereinafter, the subindex $T$ can be replaced by $H$, in order to refer to a wave plate.

The birefringence spectral dispersion, $\Delta n(\lambda)$, is a very important parameter for designing broadband photonic devices [18-21]. In this work, $\Delta n(\lambda)$ is represented using the extended Cauchy model [17], which is expressed as

$$
\Delta n(\lambda)=A_{e o}+\frac{B_{e o}}{\lambda^{2}}+\frac{C_{e o}}{\lambda^{4}},
$$

where $A_{e o}, B_{e o}$, and $C_{e o}$ are the differences between the extraordinary and ordinary Cauchy coefficients $A_{e}-A_{o}, B_{e}-B_{o}$, and $C_{e}-C_{o}$, respectively [24].

The elements $[1,1]$ and $[2,1]$ of the matrix in Eq. (1) refer to the output light polarized at $0^{\circ}$ to the $x$ axis $\left(T_{x}\right)$ and at $90^{\circ}$ to the $x$ axis $\left(T_{y}\right)$, respectively, when the input beam is $x$ polarized. 


\section{B. TN Cell between Parallel and Crossed Polarizers}

The most common display applications and characterization schemes use a TN cell between parallel and/or crossed polarizers. From Eq. (1), and considering an input beam polarized at $0^{\circ}$ to the $x$ axis, the magnitude of the transmitted beam polarized at $90^{\circ}$ to the $x$ axis, for crossed polarizers, is given by

$$
\begin{aligned}
T_{y}= & \left(\sin \left(\phi_{T}\right) \cos (X)-\frac{\phi_{T}}{X} \cos \left(\phi_{T}\right) \sin (X)\right)^{2} \\
& +\left(\frac{\Gamma_{T}}{2 X} \sin \left(2 \alpha_{T}+\phi_{T}\right) \sin (X)\right)^{2} .
\end{aligned}
$$

And, since Jones matrices are unitary, the transmitted beam polarized at $0^{\circ}$ to the $x$ axis, for parallel polarizers, can be expressed as $T_{x}=1-T_{y}$.

Equation (5) is not able to distinguish the $\phi_{T}$ sense or the difference between $\alpha_{T}$ and $\alpha_{T}+90^{\circ}$. These are called ambiguous situations. For example, using Eq. (5), in the cases where $\left[\alpha_{T}, \phi_{T}\right]=\left[0^{\circ}, 90^{\circ}\right],\left[0^{\circ}\right.$, $\left.-90^{\circ}\right],\left[90^{\circ}, 0\right]$, and $\left[90^{\circ},-90^{\circ}\right]$, the same magnitude of $T_{y}$ is obtained, and it is given by

$$
T_{y}=\cos (X)^{2}+\left(\frac{\Gamma_{T}}{2} \frac{\sin (X)}{X}\right)^{2} .
$$

For this reason, the characterization methods that use a single TN cell between parallel or crossed polarizers and Jones matrix models cannot determine a single solution for $\phi_{T}$ and $\alpha_{T}$.

\section{Characterization Method}

The proposed characterization scheme is shown in Fig. 2. Unambiguous and precise determination of TN cell parameters can be achieved simultaneously by developing a single curve-fitting procedure. It uses the spectral measurements of the transmitted beam between parallel and crossed polarizers of a reference wave plate, $\mathrm{H}$ (with well known characteristics), in series with the TN cell, at a fixed position. The wave plate is rotated at $\boldsymbol{M}$ different azimuth angles, $\alpha_{H m}$, where $m=1,2 \ldots M$. The theoretical model is obtained by using Jones calculus, and the nonlinear fitting is performed with a GA.

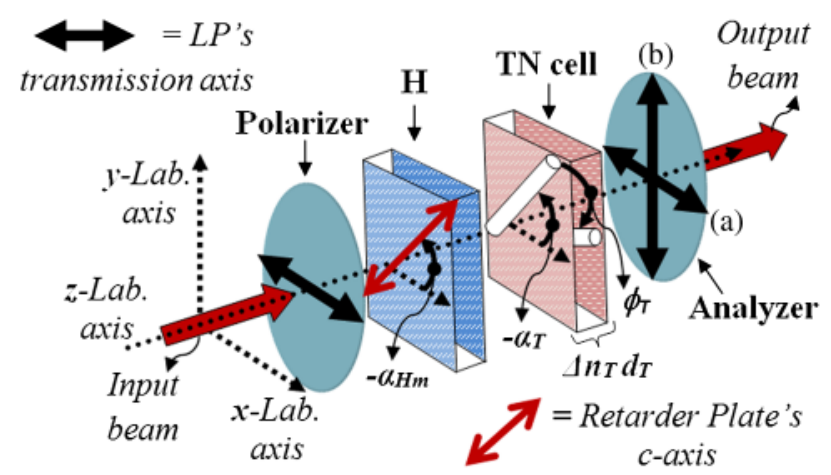

Fig. 2. Proposed characterization scheme. The angle variations in the clockwise direction are positive. (a) Parallel polarizers, (b) crossed polarizers.

\section{A. Characterization Function}

The system shown in Fig. 2 has a transfer matrix given by

$$
W=W_{T}\left(\alpha_{T}\right) W_{H}\left(\alpha_{H m}\right),
$$

where $W_{H}\left(\alpha_{H m}\right)$ is the Jones matrix of $\mathrm{H}$ :

$$
W_{H}\left(\alpha_{H m}\right)=R^{-1}\left(\alpha_{H m}\right) \times\left[\begin{array}{cc}
e^{-i \frac{\Gamma_{H}}{2}} & 0 \\
0 & e^{i \frac{\Gamma_{H}}{2}}
\end{array}\right] \times R\left(\alpha_{H m}\right) .
$$

$\Gamma_{H}$ is its phase delay [see Eq. (2)], and $R\left(\alpha_{H m}\right)$ is the rotation matrix of the angle $\alpha_{H m}$ with respect to the $x$ axis [see Eq. (3)]. Then, considering an input beam polarized at $0^{\circ}$ to the $x$ axis, the magnitude of the element $W[2,1]$ represents the transmitted beam polarized at $90^{\circ}$ to the $x$ axis:

$$
\begin{aligned}
T_{y}=\left\{\begin{array}{c}
\frac{1}{2} \cos \left(\frac{\Gamma_{H}}{2}\right)\left[\sin \left(\phi_{T}+X\right)+\sin \left(\phi_{T}-X\right)\right]+ \\
\frac{\phi_{T}}{2 X} \cos \left(\frac{\Gamma_{H}}{2}\right)\left[\sin \left(\phi_{T}-X\right)-\sin \left(\phi_{T}+X\right)\right]+ \\
\frac{\Gamma_{T}}{4 X} \sin \left(\frac{\Gamma_{H}}{2}\right)\left[\cos \left(-2 \alpha_{H m}+\sigma_{3}+X\right)\right. \\
\left.-\cos \left(-2 \alpha_{H m}+\sigma_{3}-X\right)\right]
\end{array}\right\}^{2} \\
+\left\{\begin{array}{l}
\frac{\phi_{T}}{2 X} \sin \left(\frac{\Gamma_{H}}{2}\right)\left(\sigma_{2}-\sigma_{1}\right)-\frac{1}{2} \sin \left(\frac{\Gamma_{H}}{2}\right)\left(\sigma_{1}+\sigma_{2}\right)+ \\
\frac{\Gamma_{T}}{4 X} \cos \left(\frac{\Gamma_{H}}{2}\right)\left[\cos \left(\sigma_{3}+X\right)-\cos \left(\sigma_{3}-X\right)\right]
\end{array}\right\}^{2},
\end{aligned}
$$

where $\sigma_{1}=\sin \left(2 \alpha_{H m}+\phi_{T}-X\right), \quad \sigma_{2}=\sin \left(2 \alpha_{H m}+\right.$ $\left.\phi_{T}+X\right)$, and $\sigma_{3}=2 \alpha_{T}+\phi_{T} \cdot \alpha_{T}, \alpha_{H m}$, and $\phi_{T}$ are positive in the clockwise direction, as is shown in Fig. 2. In this model it is considered that the TN cells have a zero or negligible pretilt angle.

Now, a polarization function is defined in order to relate $T_{y}$ and $T_{x}$, eliminating the influence of the losses and the ripple on the transmission spectrum of the experimental measurements for the curvefitting procedure. Since the transmission spectra are obtained for different $\alpha_{H m}$, the theoretical polarization function is defined as

$$
P_{t m}\left(\alpha_{H m}\right)=\frac{T_{y}\left(\alpha_{H m}\right)-T_{x}\left(\alpha_{H m}\right)}{T_{y}\left(\alpha_{H m}\right)+T_{x}\left(\alpha_{H m}\right)}=2 T_{y}\left(\alpha_{H m}\right)-1,
$$

where $m=1,2, \ldots M$, and the subindex $t$ indicates that it refers to the theoretical curves from Eq. (9). The polarization function $P_{t m}\left(\alpha_{H m}\right)$ varies between -1 and 1 , and it describes the preponderance of transmitted light polarized at $0^{\circ}$ to the $x$ axis over transmitted light polarized at $90^{\circ}$ to the $x$ axis. $P_{t m}\left(\alpha_{H m}\right)=-1$ means that the transmitted beam is $x$ polarized, and $P_{t m}\left(\alpha_{H m}\right)=1$ means that the transmitted beam is $y$ polarized. 
B. Minimum Azimuth Angles of $\mathrm{H}\left(\alpha_{H}\right)$ for Solving the Ambiguities

The potential of the proposed method for solving the ambiguities in the determination of TN cell parameters is analyzed by using the polarization function $P_{t m}\left(\alpha_{H m}\right)$, defined by Eq. (10). $P_{t m}\left(\alpha_{H m}\right)$ is simulated using a TN cell of $5 \mathrm{CB}$ material (with $\Delta n_{T}=0.1785$ at $589 \mathrm{~nm}$ and $25^{\circ} \mathrm{C}[24]$ ) and $d_{T}=5 \mu \mathrm{m}$, considering four ambiguous combinations of $\left[\alpha_{T}, \phi_{T}\right]\left(\left[0^{\circ}, 90^{\circ}\right]\right.$, $\left[0^{\circ},-90^{\circ}\right],\left[90^{\circ}, 0^{\circ}\right]$, and $\left.\left[90^{\circ},-90^{\circ}\right]\right)$ and a commercial wave plate $(\mathrm{H})$ of quartz $\left(\mathrm{SiO}_{2}\right)$, with $d_{H}=1 \mathrm{~mm}$, in the range from 420 to $886 \mathrm{~nm}$. The same wave plate and spectral range are used in the experimental section. Each ambiguous combination is simulated with four values of $\alpha_{H m}\left(\alpha_{H 1}=0^{\circ}, \alpha_{H 2}=-15^{\circ}, \alpha_{H 3}=-30^{\circ}\right.$, and $\alpha_{H 4}=-45^{\circ}$ ) in order to calculate the respective $P_{t m}\left(\alpha_{H m}\right)$.

Figure $\underline{3}$ shows the behavior of $P_{t m}\left(\alpha_{H m}\right)$ without including the TN cell, i.e., considering only $\mathrm{H}$ at the different values of $\alpha_{\mathrm{Hm}}$. It is observed that when the $c$ axis of $\mathrm{H}$ is at $0^{\circ}$ with respect to the $x$ axis, there is no change in the polarization state of the beam emerging from the $\mathrm{H}$ plate with regard to the beam coming from the polarizer. The maximum change in the polarization state of the beam coming from the polarizer is produced when the $c$ axis of $\mathrm{H}$ is at $-45^{\circ}$ with respect to the $x$ axis.

Figure 4 shows the behavior of $P_{t m}\left(\alpha_{H m}\right)$ for the characterization system, i.e., considering the TN cell and $\mathrm{H}$ at the four values of $\alpha_{H m}$ for each ambiguous combination of $\left[\alpha_{T}, \phi_{T}\right]$. It can be seen that $P_{t 1}\left(\alpha_{H 1}=\right.$ $\left.0^{\circ}\right)$ has the same response for the different $\left[\alpha_{T}, \phi_{T}\right]$ combinations. This case represents only the contribution of the TN cell, since $\mathrm{H}$ has no effect; therefore, Eq. (9) is equivalent to Eq. (6). On the other hand, $P_{t 4}\left(\alpha_{H 4}=-45^{\circ}\right)$ has equivalent responses for $\left[0^{\circ}\right.$, $\left.90^{\circ}\right]$ and $\left[90^{\circ},-90^{\circ}\right]$, and for $\left[0^{\circ},-90^{\circ}\right]$ and $\left[90^{\circ}\right.$, $90^{\circ}$, which represent two ambiguous situations. However, $P_{t 2}\left(\alpha_{H 2}=-15^{\circ}\right)$ and $P_{t 3}\left(\alpha_{H 3}=-30^{\circ}\right)$ have different curves for each $\left[\alpha_{T}, \phi_{T}\right]$ combination. Therefore, it can be seen that there is no ambiguity at $P_{t m}\left(\alpha_{H m}\right)$ for $0^{\circ}<\alpha_{H m}<-45^{\circ}$ in the considered spectral range.

Therefore, $P_{t 1}\left(\alpha_{H 1}=0^{\circ}\right)$ will be used to determine the parameters of the TN cell, while $P_{t 2}\left(\alpha_{H 2}=-15^{\circ}\right)$

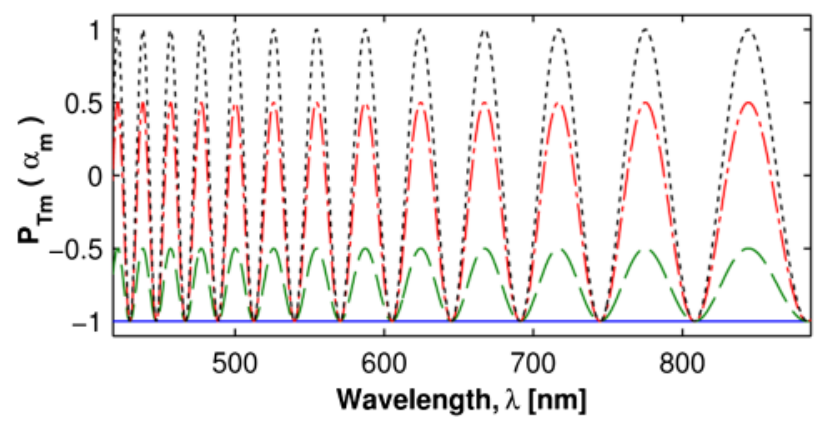

Fig. 3. Behavior of the polarization function $P_{t m}\left(\alpha_{H m}\right)$ of a wave plate $\mathrm{H}$ of quartz $\left(\mathrm{SiO}_{2}\right)$, with $d_{H}=1 \mathrm{~mm}$ and $\alpha_{H 1}=0^{\circ}$ (blue solid line), $\alpha_{H 2}=-15^{\circ}$ (green dashed line), $\alpha_{H 3}=-30^{\circ}$ (red dash-dot line), and $\alpha_{H 4}=-45^{\circ}$ (black dotted line).
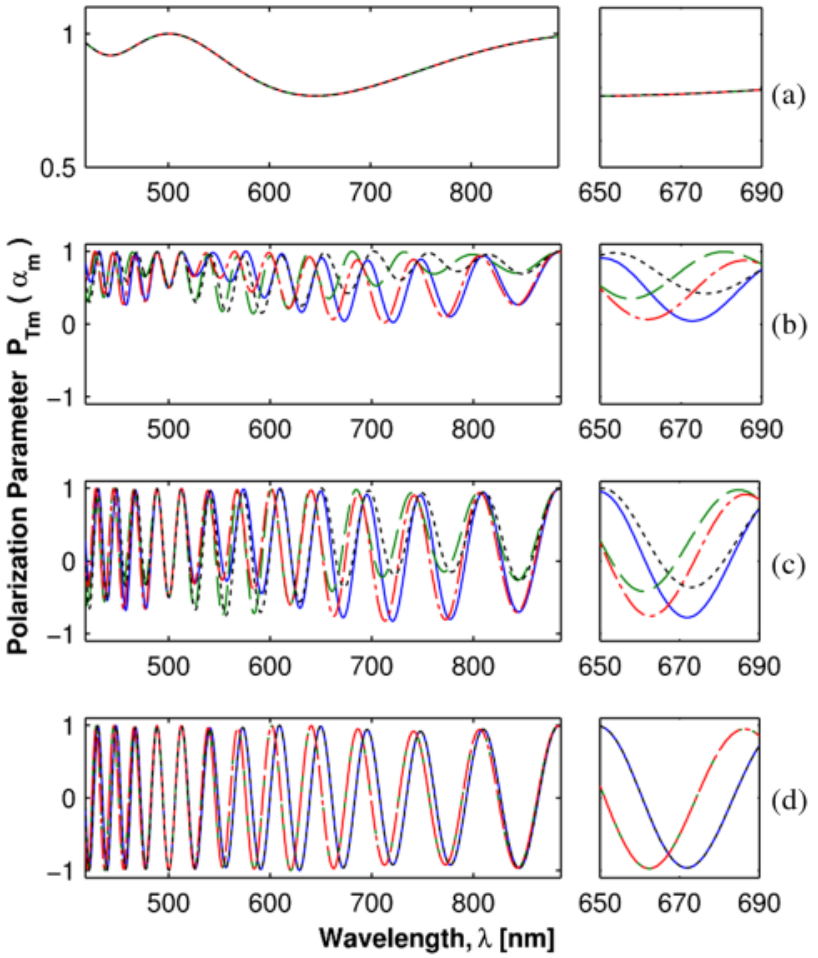

Fig. 4. Behavior of the polarization function $P_{t m}\left(\alpha_{H m}\right)$ of the proposed characterization method, considering a TN cell of 5CB and $5 \mu \mathrm{m}$ thickness with four ambiguous values of $\left[\alpha_{T}, \phi_{T}\right]=\left[0^{\circ}, 90^{\circ}\right]$ (blue solid line), $\left[0^{\circ},-90^{\circ}\right]$ (green dashed line), $\left[90^{\circ}, 90^{\circ}\right]$ (red dash-dot line), and $\left[90^{\circ},-90^{\circ}\right]$ (black dotted line). $\mathrm{H}$ is a wave plate of quartz with $d_{H}=1 \mathrm{~mm}$, and it is rotated at (a) $\alpha_{H 1}=0^{\circ}$, (b) $\alpha_{H 2}=-15^{\circ}$, (c) $\alpha_{H 3}=-30^{\circ}$, and (d) $\alpha_{H 4}=-45^{\circ}$.

and $P_{t 3}\left(\alpha_{H 3}=-30^{\circ}\right)$ will be used to eliminate the uncertainty between the ambiguous situations of $\alpha_{T}$ and $\phi_{T}$.

The phenomena shown in Fig. 4 can be described as follows. The TN cell rotates the polarization state of the incident beam $90^{\circ}$, from being $x$ polarized to being $y$ polarized, at $\sim 500$ and $\sim 900 \mathrm{~nm}$. That is why in these regions, all the ambiguous situations of Fig. $\underline{4}$ [in Figs. 4(b) $-4(\mathrm{~d})$ ] are overlapped with a value that equals the values shown in Fig. 3 at these wavelengths, but with the opposite sign. However, in the other regions, e.g., between 550 and $800 \mathrm{~nm}$, the TN cell is not able to rotate the incident light completely. Therefore, in this region we see variations in the amplitude and periodicity that are particular for each ambiguous situation, and which allow their identification. Therefore, the proposed method depends on the existence of spectral regions where the conversion efficiency of the TN cell is less than $100 \%$, which are related to the Mauguin minima. This is highly dependent on the LC cell retardation, and it is analyzed in the discussion section.

\section{Nonlinear Fitting}

The nonlinear fitting algorithm adjusts the theoretical model, given by Eq. (10), to the experimental transmittances between parallel and crossed polarizers named $T_{x e}\left(\alpha_{H m}\right)$ and $T_{y e}\left(\alpha_{H m}\right)$, respectively, 
which are obtained by using an experimental setup based on the scheme in Fig. 2. The experimental polarization function, $P_{e m}\left(\alpha_{H m}\right)$, is defined as

$$
P_{e m}\left(\alpha_{H m}\right)=\frac{T_{y e}\left(\alpha_{H m}\right)-T_{x e}\left(\alpha_{H m}\right)}{T_{y e}\left(\alpha_{H m}\right)+T_{x e}\left(\alpha_{H m}\right)} .
$$

The curve fitting can be done with any fitting algorithm. In this work it is performed by using a GA. Its random nature increments the possibility of finding a global minimum; moreover, it allows the imple- menting of the black-box function or constraints, which can be discontinuous and nondifferentiable, as part of the objective function [25]. The curve- fitting procedure considers $\alpha_{T}, \phi_{T}$, $d_{T}$, and $\Delta n(\lambda)$ as unknown variables. Therefore, the vector of fitting variables (considered as an individual by the GA) is defined as

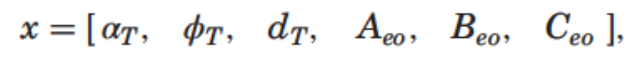

where $A_{e o}, B_{e o}$, and $C_{e o}$ are the coefficients that define $\Delta n(\lambda)$, from Eq. ( $\underline{4})$. The objective function to be minimized is given by

$$
F_{\text {obj }}(x)=\frac{1}{M} \sum_{m=1}^{M} \sqrt{\frac{1}{L} \sum_{i=1}^{L}\left[P_{e m}\left(\lambda_{i}, \alpha_{H m}\right)-P_{t m}\left(\lambda_{i}, \alpha_{H m}, x\right)\right]^{2}},
$$

where $L$ is the number of sampled wavelengths $\left(\lambda_{1}\right.$, $\left.\lambda_{2}, \ldots \lambda_{L}\right)$. This expression represents the mean value of the root mean square error (RMSE) between the theoretical and experimental values of the $M$ curves. Then, the fitting algorithm finds the optimal values of $x$ that fits the theoretical model to the experimental measurements for each $\alpha_{\mathrm{Hm}}$.

It is important to note that this method determines the LC cell optical path (retardation). Then, in order to determine $\Delta n(\lambda), d_{T}$ must be known, and vice versa.

\section{Experimental Results}

In order to demonstrate the effectiveness and simplicity of the proposed method, two experiments are presented. In each case, a different $\mathrm{TN}$ cell is characterized, and the resulting $\Delta n(\lambda)$ is compared with the respective expected value. The TN cells were manufactured with zero or negligible pretilt angle.

The experimental setup can be shown in Fig. 5 . The input beam is obtained from a halogen light source, and the transmittance measurements are taken with the spectrometer AvaSpect-128, with a spectral range from 360 to $886 \mathrm{~nm}$ and $4 \mathrm{~nm}$ of spectral resolution. Multimode optical fibers with collimators are used at the input and output ports. The input collimator produces a beam of $5 \mathrm{~mm}$ diameter. The polarizers have a diameter of $25 \mathrm{~mm}$ and an extinction ratio $>250: 1$ in the range from 420 to

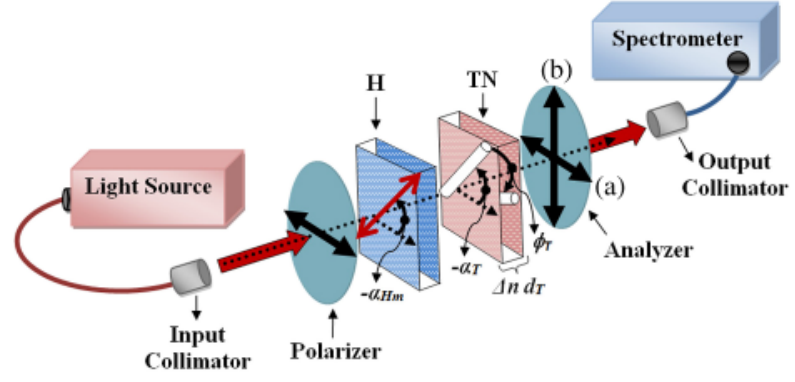

Fig. 5. Experimental setup. (a) Parallel polarizers, for $T_{x e}\left(\alpha_{H m}\right)$, and (b) crossed polarizers, for $T_{y e}\left(\alpha_{H m}\right)$. The measurements are taken at an ambient temperature of about $25^{\circ} \mathrm{C}$.

$886 \mathrm{~nm}$. The wave plate, $\mathrm{H}$, is the WPMQ05M633 , and its $\alpha_{H m}$ variations are done with a manual rotation mount at $15^{\circ}$ rotation steps. The values chosen for $\alpha_{H m}$ are $\alpha_{H 1}=0^{\circ}, \alpha_{H 2}=-15^{\circ}$, and $\alpha_{H 3}=-30^{\circ} . \alpha_{H 1}=0^{\circ}$ represents the TN cell behavior, while $\alpha_{H 2}=-15^{\circ}$ and $\alpha_{H 3}=-30^{\circ}$ allow us to identify the univocal solution of $\alpha_{T}$ and $\phi_{T}$. The transmittance curves $T_{x e}\left(\alpha_{H m}\right)$, parallel polarizers, and $T_{y e}\left(\alpha_{H m}\right)$, crossed polarizers, for $m=1,2$, and 3 are measured in the range from 420 to $886 \mathrm{~nm}$ (limited by the polarizers and spectrometer operation ranges).

Once $T_{x e}\left(\alpha_{H m}\right)$ and $T_{y e}\left(\alpha_{H m}\right)$ are obtained, the experimental polarization function $P_{e m}\left(\alpha_{H m}\right)$ is calculated, for $m=1,2$, and 3 , and the curve-fitting procedure is performed. The population size is set to 1,000 (number of $x$ combinations per iteration), and the maximum number of iterations is set to 100 . Wide search limits are set for $\alpha_{T}, \phi_{T}$, and $\Delta n(\lambda) \quad\left(-110^{\circ} \leq \alpha_{T} \leq 110^{\circ}, \quad 80^{\circ} \leq\left|\phi_{T}\right| \leq 100^{\circ}\right.$, $0 \leq A_{e o} \leq 0.4, \quad 0 \leq B_{e o} \leq 0.03$, and $0 \leq C_{e o} \leq 0.03$ [24]), and all the initial conditions are set to 0 , in order to demonstrate the convergence capacity of the proposed method. $d_{T}$ is fixed to its respective theoretical value (for each characterized TN cell), since $\Delta n(\lambda)$ has been set as a variable.

\section{A. First Experiment}

A TN cell made with 5CB LC material, $90^{\circ}$ twist, and $5.1 \mu \mathrm{m}$ thickness (named TN1) is characterized.

Figure $\underline{6}$ shows the curves of the polarization function $P_{e m}\left(\alpha_{H m}\right)$ for the three different values of $\alpha_{H m}$ (dots). From these data, the nonlinear fitting procedure finds the optimal theoretical model for TN1, which is defined by: $\alpha_{T 1}=82.6^{\circ}, \phi_{T 1}=$ $-87.1^{\circ}, d_{T 1}=5.1 \mu \mathrm{m}, A_{e o T 1}=0.1605, B_{e o T 1}=0.0011$, and $C_{e o T 1}=0.0017$. The curve fitting results are also shown in Fig. 6 (solid lines), where $e_{r r m}$ represents the RMSE between the experimental and theoretical curves.

In Fig. 7 , the resulting birefringence of TN1, $\Delta n_{T 1}(\lambda)$, which is defined by $A_{e o T 1}=0.1605$, $B_{e o T 1}=0.0011$, and $C_{e o T 1}=0.0017$ (solid line), is compared with the birefringence of the 5CB LC material, taken from [24], which is defined by $A_{e o}=0.1569, B_{e o}=0.0029$, and $C_{e o}=0.0016$ (dots). 


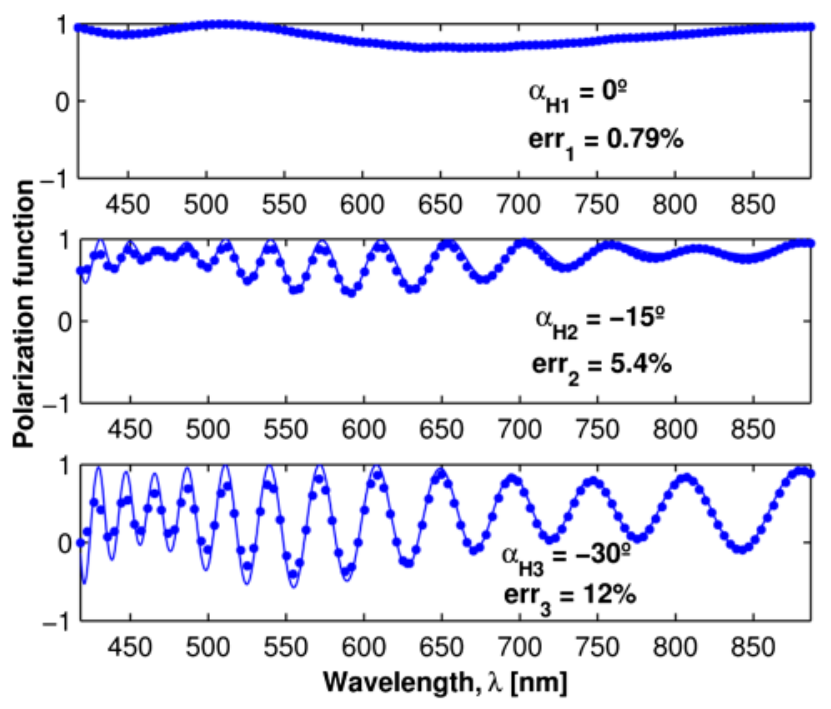

Fig. 6. Polarization function of the TN cell TN1 in series with $\mathrm{H}$. Solid lines represent the curve fitting, $P_{t m}\left(\alpha_{H m}\right)$, and the dots represent the experimental measurements, $P_{e m}\left(\alpha_{H m}\right)$.

The maximum relative error between both curves is less than $1 \%$ in the range from 420 to $886 \mathrm{~nm}$.

\section{B. Second Experiment}

A TN cell made with $1658 \mathrm{LC}, 90^{\circ}$ twist, and $5.1 \mu \mathrm{m}$ thickness (named TN2) is characterized. This is an experimental LC material synthesized at the Military University of Technology in Warsaw.

Figure $\frac{8}{P}$ shows the experimental polarization function $\bar{P}_{e m}\left(\alpha_{H m}\right)$ for the three different values of $\alpha_{H m}$ (dots). The theoretical model responses are also plotted (solid lines). This model is obtained with the nonlinear fitting algorithm, and it is defined by

$\alpha_{T 2}=86.2^{\circ}, \phi_{T 2}=91.4^{\circ}, d_{T 2}=5.1 \mu \mathrm{m}, A_{\text {eo T2 }}=$ $0.3103, B_{e o T 2}=0$, and $C_{e o T 2}=0.0063$.

In Fig. 9 , the resulting birefringence of TN2, $\Delta n_{T 2}(\lambda)$, which is defined by $A_{e o T 2}=0.3103, B_{e o T 2}=$ 0 , and $C_{e o T 2}=0.0063$ (solid line), is com- pared with the birefringence values of the $1658 \mathrm{LC}$ material measured at six different wavelengths (dots). These values were obtained for cells of the same material, using a different characterization method [26]. The validity of these data was tested in a previous work [20], and the points are limited between 520 and 886 nm due to the LPs and the

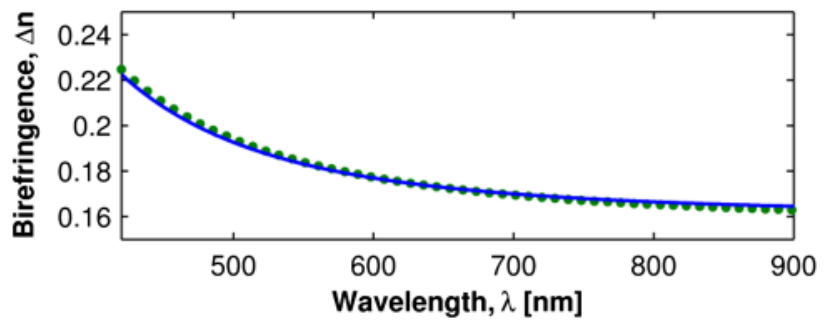

Fig. 7. Birefringence of the TN cell TN1, defined by $A_{e o T 1}=0.1605, B_{e o T 1}=0.0011$, and $C_{e o T 1}=0.0017$ (solid line), and the theoretical birefringence of the 5CB LC material, defined by $A_{e o}=0.1569, B_{e o}=0.0029$, and $C_{e o}=0.0016$ (dots).

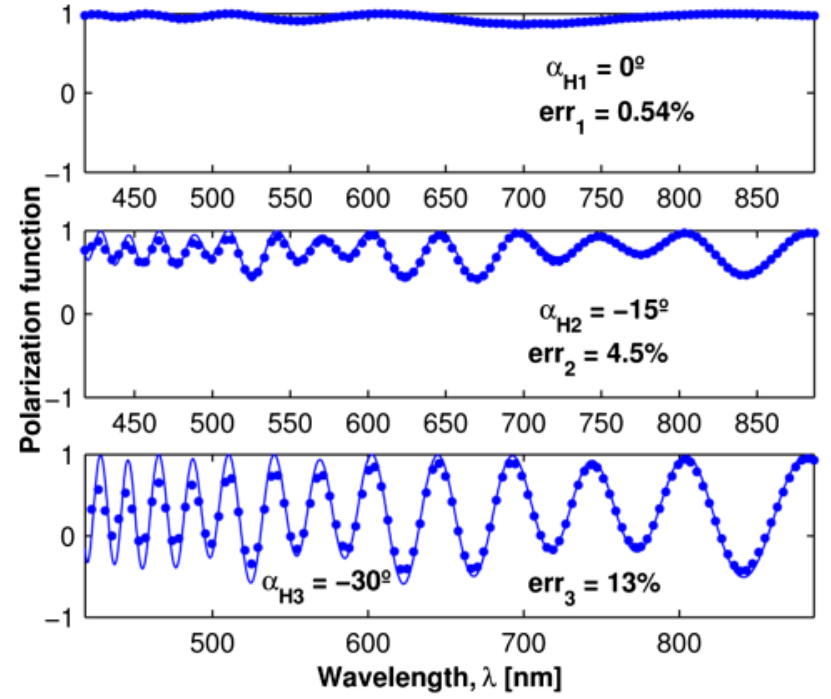

Fig. 8. Polarization function of the TN cell TN2 in series with $\mathrm{H}$. Solid lines represent the curve fitting, $P_{t m}\left(\alpha_{H m}\right)$, and the dots represent experimental measurements, $P_{e m}\left(\alpha_{H m}\right)$.

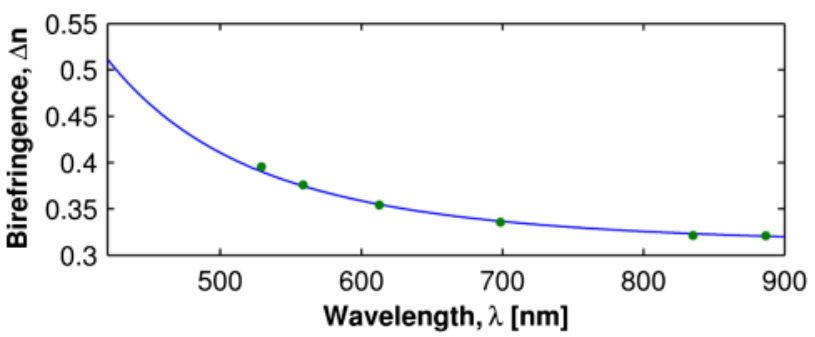

Fig. 9. Birefringence of the TN cell TN2, defined by $A_{\text {eoT2 }}=0.3103, \quad B_{\text {eoT2 }}=0, \quad$ and $C_{\text {eoT2 }}=0.0063$ (solid line), and the expected birefringence values of the LC-1658 material at various wavelengths (dots) obtained from a homogeneous cell by a different characterization method.

spectrometer used in their characterization [20]. The relative error between $\Delta n_{T 2}(\lambda)$ and the previous characterized values $[20,26]$ is less than $1.3 \%$.

\section{Discussion}

The proposed method allows us to unambiguously determine the most important parameters of TN cells, unlike other spectral methods. And unlike single wavelength methods, it can also accurately determine the LC birefringence spectral dispersion, $\Delta n(\lambda)$.

It has been shown that only six measurements are required, since a spectrometer can simultaneously measure all the wavelengths, unlike a polarimeter or a single detector. The smaller number of measurements decreases the error sources.

The system uses manual rotation mounts and a low-resolution spectrometer. Therefore, the proposed method can be simpler and cheaper than those using high-resolution motorized rotation stages, polarimeters, and monochromatic light sources.

The simple approach of using a Jones matrix has allowed us to design a fast fitting algorithm. The 
previous section results were obtained using a population of 1000 individuals (number of combinations of $x$ per iteration) and 116 wavelengths (spectral range from 420 to 886 $\mathrm{nm}$ with a $4 \mathrm{~nm}$ resolution). Under these conditions, the average convergence time was $20^{\prime \prime}$ (35 iterations on average) using a computer with CPU Intel Core 2 Duo at $2.66 \mathrm{GHz}$ and RAM of $3.25 \mathrm{~GB}$. The search time can be reduced to 12 " using a spectral resolution of $8 \mathrm{~nm} \mathrm{(58}$ wavelengths), with very similar results to those obtained in the previous section.

It is important to note that $e_{r r 3}>e_{r r 2}>e_{r r 1}$ in Figs. $\underline{6}$ and $\underline{8}$. This is because the amplitude of the

peaks of $P_{e m}\left(\alpha_{H m}\right)$ increases as $\alpha_{H m}$ approaches $\pm 45^{\circ}$, and therefore a better sampling resolution is required. However, this does not affect the calculation of the cell parameters. The adjustment of the curve $P_{e 1}\left(\alpha_{H 1}=0^{\circ}\right)$, given by $e_{r r 1}$, defines the accuracy in the calculation of the cell parameters, since there is no influence of $\mathrm{H}$, as is demonstrated in the birefringence results (Figs. $\underline{7}$ and $\underline{9}$ ).

The adjustment of the curves $P_{e 2}\left(\alpha_{H 2}=-15^{\circ}\right)$ and $P_{e 3}\left(\alpha_{H 2}=-30^{\circ}\right)$, given by $e_{r r 2}$ and $e_{r r 3}$, defines the uniqueness of $\alpha_{T}$ and $\phi_{T}$. For example, the solutions

of $\alpha_{T}$ and $\phi_{T}$ of cell TN1 are $\alpha_{T 1}=82.6^{\circ}$ and $\phi_{T 1}=$ $-87.1^{\circ}$ with $e_{r r 1}=0.79 \%, \quad e_{r r 2}=5.4 \%$, and $e_{r r 3}=12 \%$; however, if we replace $\alpha_{T 1}$ by $\alpha_{T 1}+90^{\circ}$, the same value of $e_{r r 1}$ is obtained, but the values of $e_{r r 2}$ and $e_{r r 3}$ change to $23 \%$ and $40 \%$, respectively.

The search limits of $\alpha_{T}$ and $\Delta n(\lambda)$ are very wide. This allows us to characterize high-birefringence LC cells, such as those made of 1658 LC material. This method can obtain the unique solution for the $\alpha_{T}$ and $\phi_{T}$ of TN cells with retardations between 0.91 and $1.85 \mu \mathrm{m}$; e.g., TN1 has retardation $=5.1 \mu \mathrm{m} \times$ 0.1786 (at $589 \mathrm{~nm}$ and $\sim 25^{\circ} \mathrm{C}$ ) $\approx 0.91$, and $\mathrm{TN} 2$ has retardation $=5.1 \mu \mathrm{m} \times 0.3625$ (at $589 \mathrm{~nm}$ and $\sim 25^{\circ}$ C) $\approx 1.85$. This is described in the next sub- section. $\phi_{T}$ limits were set as $80^{\circ} \leq|\phi| \leq 100^{\circ}$, since it was known that the cells were twisted $90^{\circ}$. This range is large in comparison with those reported in [13], of $90^{\circ} \pm 5^{\circ}$. However, different tests have been performed with larger limits on $\phi$ (even with

$\left.-360^{\circ} \leq \phi \leq 360^{\circ}\right)$, by using $\alpha_{H}=0^{\circ},-15^{\circ},-30^{\circ}$, and $-45^{\circ}$, with an average convergence time of $24^{\prime \prime}$. These limits include cells with arbitrary twists, even super-twisted cells $\left(\left|\phi_{T}\right|=270^{\circ}\right)$.

Other tests were performed using wave plates of low order, e.g., a nematic homogeneous cell of 5CB and $5 \mu \mathrm{m}$. In these cases there is better curve fitting (less error between curves), but with slower convergence (more iterations) and with less accurate results. All the tests done with this method, including the results of the previous section, were performed using experimental LC cells.

The results of $\Delta n_{T 1}(\lambda)$ and $\Delta n_{T 2}(\lambda)$ have relative errors less than $1.3 \%$, with respect to the theoretical values, which demonstrates the effectiveness and accuracy of the method. In general, the possible difference between $\Delta n$ or $d_{T}$ and their theoretical values are given by other characteristics of the cell, such as the pretilt angle, the dopants (for twist purpose), or the temperature effects.

The proposed method does not consider the pretilt angle of the cells, so it is designed to characterize TN cells with zero or negligible pretilt angle. However, the pretilt angle can also be included in the model, considering its effects on the variation of the effective birefringence [27].

\section{A. Retardation Range That Can be Characterized Unambiguously}

In order to demonstrate that the proposed method can distinguish between the possible ambiguous situations of $\alpha_{T}$ and $\phi_{T}$ in TN cells with wide retardation ranges, it is important to know how different the curves of $P_{t 2}\left(\alpha_{H 2}=-15^{\circ}\right)$ and $P_{t 3}\left(\alpha_{H 3}=-30^{\circ}\right)$ are, produced by the different combinations of $\left[\alpha_{T}, \phi_{T}\right]$. A new parameter is defined to estimate the degree of differentiation, $\Delta P_{\min }$. It is obtained by subtracting the different curves of Figs. $\underline{4(b)}$ and $\underline{4(c)}$, two by two, and then the root mean square (RMS) of each subtraction is calculated and the minimum RMS value is selected. The maximum possible value of $\Delta P_{\min }$ is 2 . If $\Delta P_{\min }=2$, the curves produced by the ambiguous combinations are $100 \%$ different. On the other hand, if $\Delta P_{\min }=0$, the curves produced by the ambiguous combinations are equivalent. Then, $\Delta P_{\min }$ defines the ability of the proposed characterization method to identify the unique answer of the parameters of an unknown TN cell at a specific $\alpha_{\mathrm{Hm}}$.

The curves of $P_{t 2}\left(\alpha_{H 2}=-15^{\circ}\right)$ and $P_{t 3}\left(\alpha_{H 3}=-30^{\circ}\right)$, produced under the different combinations of $\left[\alpha_{T}, \phi_{T}\right]$ shown in Fig. 4, are obtained by simulating a TN cell of $d_{T}=5 \mu \mathrm{m}$ and 5CB material, with a retardation of $5 \mu \mathrm{m} \times 0.1786$ (at $589 \mathrm{~nm}$ and $\sim 25^{\circ} \mathrm{C}$ ) $\approx 0.89 \mu \mathrm{m}$. In this case, the resulting $\Delta P_{\min }$ of $P_{t 2}\left(\alpha_{H 2}=-15^{\circ}\right)$ and $P_{t 3}\left(\alpha_{H 3}=-30^{\circ}\right)$ is about 0.28 . In order to determine the minimum and maximum retardation of the TN cells that can be characterized with the proposed system, Fig. 10 shows the variation of $\Delta P_{\min }$ for values of $d_{T}$ between 0.5 and $11 \mu \mathrm{m}$, which represent retardations between $\sim 0.09$ and $\sim 1.96 \mu \mathrm{m}$ for cells of $5 \mathrm{CB}$ at $589 \mathrm{~nm}$ at $25^{\circ} \mathrm{C}$, under the same conditions as the simulations presented in Fig. 4, for $\alpha_{H 2}=-15^{\circ}$ (solid line) and $\alpha_{H 3}=-30^{\circ}$ (dashed line). It can be seen that $\Delta P_{\min }>0.13$ in the whole considered

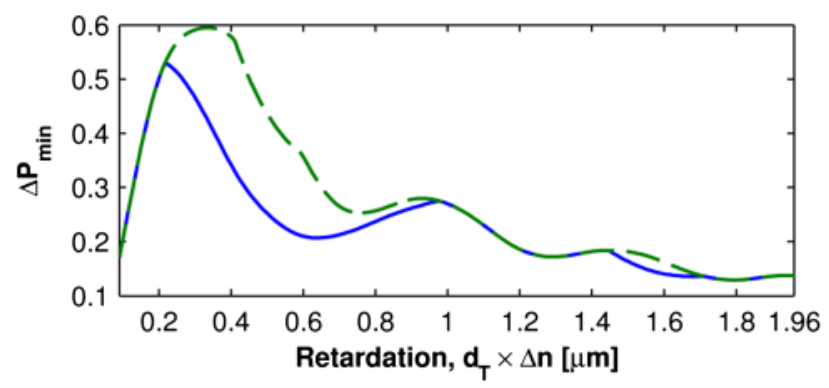

Fig. 10. $\Delta P_{\min }$ of the curves of $P_{t 2}\left(\alpha_{H 2}=-15^{\circ}\right)$ (solid line) and $P_{t 3}\left(\alpha_{H 3}=-30^{\circ}\right)$ (dashed line) produced under the different combinations of $\left[\alpha_{T}, \phi_{T}\right]$ of a TN cell of 5CB material using the proposed characterization method. 
range, which includes very thin cells [14]. $\Delta P_{\min }>$ 0.13 is sufficient to identify the unique solution for the parameters $\left(\alpha_{T}, \phi_{T}\right)$ of an unknown TN cell by using the proposed method. This is demonstrated in the experimental section by characterizing a TN cell of $1658 \mathrm{LC}$ (TN2), with retardation of about 1.85 $\mu \mathrm{m}\left(\Delta P_{\min } \approx 0.13\right.$; see Fig. 10$)$. Cells with more retardation can be characterized using more azimuth angles, $\alpha_{\mathrm{Hm}}$.

It is important to note that Eq. (9) is not mathematically complicated, as it keeps the simplicity of using a Jones matrix and solves the ambiguities of Eq. (ㅁ) without the need to perform additional measurements or analysis.

\section{Conclusion}

We have proposed a simple experimental method for univocal determination of nematic LC cell parameters. Unlike previous proposals, it uses few angle variations, uses wide searching limits, needs no initial conditions, and can be used for determining the spectral dispersion of the LC birefringence. It is ideal for broadband applications.

The characterization results of two different TN cells have been presented (TN1 and TN2). The results of their physical parameters $\left(\alpha_{T 1}, \alpha_{T 2}, \phi_{T 1}\right.$, and $\left.\phi_{T 2}\right)$ and birefringence $\left[\Delta n_{T 1}(\lambda)\right.$ and $\left.\Delta n_{T 2}(\lambda)\right]$ are consistent with the theoretical expectations. The measured $\Delta n_{T 1}(\lambda)$ has a relative error of less than $1 \%$ with respect to the 5CB LC birefringence in the range from 420 to $890 \mathrm{~nm}$, and $\Delta n_{T 2}(\lambda)$ has a relative error of less than $1.3 \%$ with respect to a previous characterization with a different method of the 1658 LC material.

The proposed characterization method can also be applied to characterizing homogeneous nematic devices, which are widely used in the design of tunable optical filters $[19,20]$. It can be easily package in a precise, fast, and automatic characterization system.

The authors acknowledge the Military University of Technology (MUT/WAT) in Warsaw and the Polytechnic University of Madrid (CEMDATIC UPMfor supplying the LC devices. This work was supported in part by the Ministerio de Economía y Competitividad of Spain under Grant TEC2012-37 983-C03-02.

\section{References}

1. J. Beeckman, K. Neyts, and P. J. M. Vanbrabant, "Liquidcrystal photonic applications," Opt. Eng. 50, 081202 (2011).

2. C. Vázquez, I. Pérez, P. Contreras, B. Vinouze, and B. Fracasso, "Liquid crystal optical switches," in Optical Switches-Materials and Design, B. Li and S. J. Chua, eds. (Woodhead, 2010), Chap. 8.

3. P. Yeh and C. Gu, Optics of Liquid Crystal Displays (Wiley, 2010).

4. P. Contreras, "Advanced devices based on fibers, integrated optics and liquid crystals for WDM networks," Ph.D. thesis (Universidad Carlos III de Madrid, May 2011), http:// e-archivo.uc3m.es/handle/10016/11587.

5. P. C. Lallana, C. Vázquez, and B. Vinouze, "Advanced multifunctional optical switch for multimode optical fiber networks," Opt. Commun. 285, 2802-2808 (2012).
6. C. Soutar and K. Lu, "Determination of the physical properties of an arbitrary twisted nematic liquid crystal cell," Opt. Eng. 33, 2704-2712 (1994).

7. HOLOEYE Photonics AG, "OptiXplorer Manual," 2007, http:/ www.pa.msu.edu/courses/2011fall/PHY431/Labs/Optixplorer_ Manual_v2.8f_sec.pdf.

8. J. A. Davis, I. Moreno, D. B. Allison, K. G. D'Nelly, and M. L. Wilson, "Ambiguities in measuring the physical parameters for twisted-nematic liquid crystal spatial light modulators," Opt. Eng. 38, 705-709 (1999).

9. H. Kim and Y. H. Lee, "Unique measurement of the parameters of a twisted-nematic liquid-crystal display," Appl. Opt. 44, 1642-1649 (2005).

10. V. Durán, J. Lancis, and E. Tajahuerce, "Cell parameter determination of a twisted-nematic liquid crystal display by single-wavelength polarimetry," J. Appl. Phys. 97, 043101 (2005).

11. C. Yu, Y. Tseng, K. Hsu, and C. Chou, "Analytical approach for unique determination of cell parameters and equivalent birefringent parameters of a generally twisted nematic liquid crystal device," Appl. Opt. 51, 7910-7919 (2012).

12. W. Hung, M. Tsai, C. Wang, I. Jiang, and W. Cheng, "Simple parameter determination for twisted nematic liquid-crystal display," Appl. Opt. 46, 3493-3497 (2007).

13. Y. Lo, Y. Chung, C. Liao, and W. Hsieh, "Transmitted ellipsometry method for extracting physical parameters of TN/VA/Inverse-TN liquid crystal cells," IEEE J. Quantum Electron. 49, 259-266 (2013).

14. S. Valyukh, I. Valyukh, and K. Skarp, "Spectrophotometric determination of reflective liquid crystal cell parameters," J. Appl. Phys. 99, 053102 (2006).

15. R. Giust and J. P. Goedgebuer, "Determination of the twist angle and the retardation properties of twisted nematic liquid crystal television by spectral measurements," Opt. Eng. 37, 629-634 (1998).

16. S. T. Tang and H. S. Kwok, "Transmissive liquid crystal cell parameters measurement by spectroscopic ellipsometry," J. Appl. Phys. 89, 80-85 (2001).

17. J. S. Chae and S. G. Moon, "Cell parameter measurement of a twisted-nematic liquid crystal cell by the spectroscopic method," J. Appl. Phys. 95, 3250-3254 (2004).

18. Q. Wang, G. Farrell, T. Freir, and J. She, "Optimal design of broadband linear polarization converters/switches," J. Opt. A Pure Appl. Opt. 7, 47-50 (2005).

19. O. Aharon and I. Abdulhalim, "Liquid crystal Lyot tunable filter with extended free spectral range," Opt. Express 17, 11426-11433 (2009).

20. P. J. Pinzón, I. Pérez, C. Vázquez, and J. M. Sánchez Pena, "Reconfigurable $1 \times 2$ wavelength selective switch using high birefringence nematic liquid crystals," Appl. Opt. 51, 5960-5965 (2012).

21. P. J. Pinzón, C. Vázquez, I. Pérez, and J. M. Sánchez Pena, "Synthesis of asymmetric flat-top birefringent interleaver based on digital filter design and genetic algorithm," IEEE Photon. J. 5, 7100113 (2013).

22. D. A. Yakovlev, V. G. Chigrinov, and H. S. Kwok, Modeling and Optimization of LCD Optical Performance (Wiley, 2014).

23. B. E. A. Saleh and K. Lu, "Theory and design of the liquid crystal TV as an optical spatial phase modulator," Opt. Eng. 29, 240-246 (1990).

24. J. Li, C. Wen, S. Gauza, R. Lu, and S. Wu, "Refractive indices of liquid crystals for display applications," J. Disp. Technol. 1, 51-61 (2005).

25. Global Optimization Toolbox, MatWorks, Natick, MA, 2012, http://www.mathworks.com/products/datasheets/pdf/globaloptimization-toolbox.pdf.

26. V. Urruchi, I. Pérez, N. Ganoa, and J. M. Sánches-Pena, "Phase modulation and optical anisotropy of high birefringence liquid crystals," Mol. Cryst. Liq. Cryst. 502, 207-219 (2009).

27. V. Belyaev, A. Solomatin, and D. Chausov, "Phase retardation vs. pretilt angle in liquid crystal cells with homogeneous and inhomogeneous LC director configuration,” Opt. Express 21, 4244-4249 (2013). 